\title{
The Use of CRISPR-Cas9 Technology to Study and Create Cancer Therapeutics
}

\author{
Andrew J. Kennedy and Jeffrey O. Henderson ${ }^{\mathrm{a}}$
}

Genetic disorders are the result of abnormalities that arise in the human genome at birth or through postnatal random genetic change. These abnormalities can also increase the risk for developing other diseases such as cancerous cell growth. Traditional treatment for genetic disorders has focused on alleviation of symptoms to increase patient welfare rather than treating the root cause, the genetic abnormality. As genetic editing technologies are developed and refined, the prospect of correcting the abnormal genetic sequence is becoming realistic. The CRISPR-Cas9 system has made it possible for researchers to respond to genetic abnormalities quickly by cutting and replacing the abnormal sequence to then contain a healthy sequence and potentially reverse the abnormal phenotype. Cancer, a disease based on genetic dysfunction, is a prime target for genetic editing. Often treated with debilitating radiation, chemotherapy, or surgery, the use of genetic editing has the potential to revolutionize current treatment options. This review will discuss the current outlook of cancer and its treatment with a focus on how CRISPR-Cas9 can be used to edit immunotherapy options that clinicians currently possess. Furthermore, potential dangers of the CRISPR-Cas9 technology and consequences of the system and its unethical use will be discussed. Finally, there will be an evaluation on the future of how CRISPR-Cas 9 can be used in medicine.

Keywords: CRISPR-Cas9, Cancer, Genetic Editing, Bioethics, Biotechnology

With the advent of modern biotechnology, there are new approaches to the world's biomedical problems. Genetic editing has recently been developed to correct aberrant genomic sequences which cause disorder or predispose patients to increased risk of disease. A technology of recent focus has been the Clustered Regularly Interspaced Short Palindromic Repeats (CRISPR) coupled with a CRISPR Associated Protein 9 (Cas9) creating the CRISPR-Cas9 system. The technology has been lauded for its cost, ease of use, and specificity for the intended sequence. The CRISPR-Cas 9 system works by cutting out or replacing sequences to correct abnormalities within a patients DNA. The system scientists are developing is originally based on the immune system in the archaeal salttolerant microbe Haloferex mediterranai, first identified by the Rodriguez-Valera lab in Alicante, Spain in 1993 (Mojica et al., 1995). This small microbe was later found to contain a genetic editing system that opened the possibility to edit the human genome, something the Rodríguez-Valera lab did not originally predict.

In the past 15 years, the development of CRISPR-Cas9 has been monumental within biotechnology. Starting in 2006, the system was proposed as an immune response by prokaryotes using small-RNA based changes to "remember" previous pathogens such as bacteria and viruses (Chen et al., 2016). Marraffini and Sontheimer (2008) then demonstrated that the system acts as a target of DNA, not RNA, and could artificially move from bacteria to other organisms, opening the possibility of its use in humans. In previous attempts at genetic editing with Zinc Finger Nucleases (ZFNs) and Transcription Activator-Like Effector Nucleases (TALENs), one of the main problems was that edits were made at an unintended place in the genome (Cho et al., 2013). These off-target effects result from inconsistent targeting by the molecule created to "seek out" the sequence. In contrast, Garneau and coworkers (2010) discovered that the Cas9 nuclease cuts the target DNA with high specificity through the use of only the Cas9 and a three nucleotide sequence upstream of the target named the Protospacer Adjacent Motif (PAM). The next advance was the development of a synthetic single guide RNA (sgRNA) used to attach to the sequence. The sgRNA is developed by selection of a crispr RNA (crRNA) corresponding to the sequence of interest which is then fused with a trans-activating RNA (tracrRNA) which acts in tandem as a single molecule assisting the Cas 9 molecule in finding the sequence (Jinek et al., 2013). Researchers then, in late 2013, demonstrated the editing of eukaryotic cells, expanding the number of organisms that CRISPR-Cas9 could be used in (Cong et al., 2013). As a result of these advances, CRISPR-Cas9 became a reality for custom genetic editing within organisms that do not naturally possess this ability.

The excitement surrounding CRISPR-Cas9 is merited; but equally important to clarify is the realistic potential it has in fixing genetic abnormalities in the population. Researchers are heavily interested in the system's potential to precisely cut a sequence to the specified start and end nucleotide of interest (Kidiyoor, 2018). Furthermore, researchers consider the system easy to use, a cheaper alternative to previous gene editing technologies, and application of the research has been successful in early trials with mice (Ledford, 2015). The prospect of cheap, efficient, and long lasting genetic editing is exciting as healthcare remains the emphasis for the future, especially as a response to cancer, a disorder based on genetic abnormalities (Mullin, 2018).

\section{Mechanism of CRISPR-Cas9}

The CRISPR-Cas9 system pairs biotechnological innovation with a naturally occurring bacterial defense system. The process (Figure 1) begins with a single guide RNA (sgRNA), the result of combining two smaller molecules, the crisprRNA (crRNA) and the trans-activating crRNA (tracrRNA). This forms a duplex which attaches to the sequence of interest (Jinek et al., 2013). The sequence of interest lies next to a protospacer adjacent motif (PAM) sequence, an NAG or NGG trinucleotide, immediately upstream of the intended edit (Hsu et al., 2013). The corresponding sequence is then cut by the Cas9 nuclease inducing a double strand break (Mali et al., 2013). Once the Cas9 nuclease induces the double strand break, the intended 
edits are accomplished through a repair of the broken sequence. This is done through nonhomologous end-joining (NHEJ), with the break being repaired without inserting a new sequence, or homology-directed repair (HDR), with the intended sequence being inserted, after which the edit is then complete. The specified sequence inserted is created in the lab and then transformed into the cell in the same way the sgRNA and Cas9 nuclease are inserted (Griffiths et al., 1999). After this process is finished, the cell will continue dividing, with the edits being permanent and carrying on to the next generation, in turn completing the edit process.

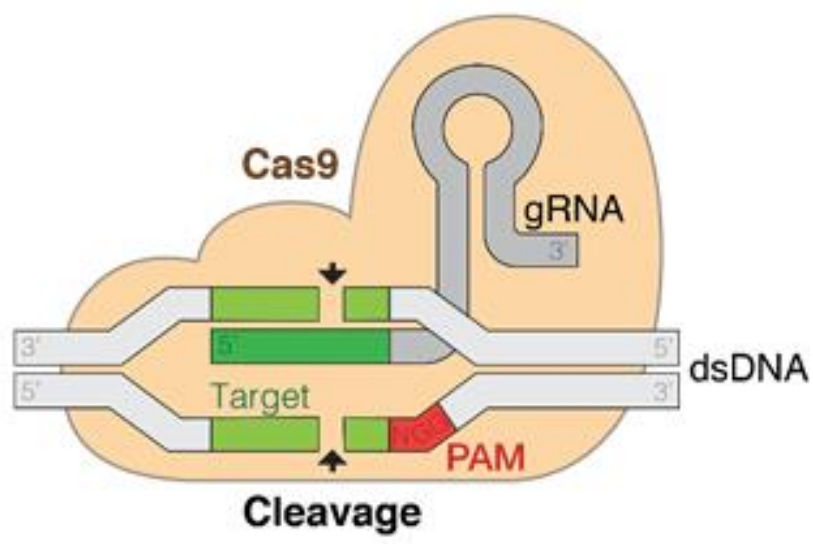

Figure 1. Model of CRISPR-Cas9 Activity. Artist (Walter, 2017) rendition of the Cas 9 complex including the PAM sequence lying upstream of the intended sequence for editing. This image also shows the gRNA which specifies the sequence which needs to be cut.

\section{Failure of CRISPR-Cas9 to Work}

Though the CRISPR-Cas9 system has numerous benefits there are still issues with integrating the technology into DNA which keeps it from current use. The largest impediment lies in determining proper delivery of the sgRNA and Cas9 nuclease to the intended cell. Delivery is often attempted using a viral vector, although this has been shown to produce integration problems due to the body's immune response to the viral particles (Kidiyoor, 2018). Furthermore, integrating edits into brain cells can be tough as the blood brain barrier often prevents molecules from passing through (Geldenhuys et al., 2015). Lastly, it can be a challenge to edit quickly replicating cells, as researchers must ensure the change occurs before cell division. If cell division occurs before the edit is complete, coding sequences may be negatively affected.

Researchers have recently observed an immunologic response to components of the CRISPR system though they are still not able to predict the unique immune response a patient will have against many foreign or self-antigens (Kidiyoor, 2018). Though responses to common pathogens and chemicals are known, using a molecule such as Cas9 or the sgRNA could potentially produce an adverse response we cannot predict (Chaplin, 2003). The bacteria Streptococcus pyogenes and Streptococcus aureus are the original organisms from which CRISPR components were isolated from, with products from these organisms being maintained into editing. However, according to new data, researchers have found antibodies in patients that occur naturally in the body against these organisms (Charlesworth et al., 2018). This immunity predisposes the body against the vector for delivery. It follows that if the editing mechanism is a constituent of one of these organisms, the body will attack the foreign molecule due to predisposed immunity. Charlesworth and coworkers (2018) also observed that during editing, any molecules containing Cas9 peptides were destroyed by the immune system, stopping any eventual CRISPR activity. There is currently only limited clinical data on how to effectively deliver the CRISPR system without editing halting due to immune response or failure to insert into the cell. Li and coworkers (2015) have demonstrated that in vitro methods are, so far, showing potential for editing efficiency with Charlesworth and coworkers (2018) reporting that the immune system of the patient's body does not interfere with the editing process.. However, it has still been suggested that patients with preexisting immunity against components of the system be left out of clinical trials as determining efficacy and safety may be skewed by this population (Kidiyoor, 2018).

Off-target binding continues to be a problem in CRISPRCas9 genetic editing. Numerous target sequences are common across the genome, potentially interfering with the specificity of sgRNA binding to the intended sequence (Macdonald et al., 2004). This interference can eventually lead to off target binding as the sgRNA will bind to a highly similar, yet still incorrect, sequence being cut or inserted and causing further problems. The problems with binding deal mainly with the interactions between Cas9 and sgRNA. Specifically, "Cas9 nucleases (are) complexed with three sgRNAs induc(ing) offtarget mutations at six sites that differ by 1 or $2 \mathrm{nt}$ from their corresponding on target sites with frequencies that ranged from $0.5 \%$ to $10 \%$." It was also found that when the Cas 9 was paired with another Cas9 the rate of spontaneous insertions or deletions dropped below the detection limit of $0.1 \%$ (Cho et al., 2013). Off target effects could have grave risks if the sequence lies in a physiologically important area such as for the coding of a hormone or protein.

As mentioned before, a protospacer adjacent motif is required for recognition of the target sequence. The targeting of some sequences is not possible due to the PAM being absent with the sgRNA having no sequence to bind to before cutting (Agtmaal et al., 2017). Many sequences do in fact have a PAM sequence; the sequence of interest needs to simply have multiple NAG or NGG trinucleotides upstream of the edit, a common characteristic in the genome (Ran et al., 2013). The number of intended sequences lacking a PAM is likely minimal but the absence of a PAM sequence in any sequence of interest hinders the use of the CRISPR-Cas9 system as a whole.

\section{Medical Aspects of CRISPR-Cas9 Not Working}

Genetic editing seeks to permanently change the genome of a patient meaning that the "drug" cannot simply be withdrawn from a patient's medication list. The permanent nature of this therapy demands that safety be assured before moving into human clinical trials. No matter what, Reardon (2016), in a review in Nature News, believes a "leap of faith" has to be made as researchers move into human trials. Clinical trial schedules for traditional drugs allow for the drug of study to be discontinued and the effects of the drug likely dissipating. However, as genetic edits are made, it can be hard to move into in vivo testing before ensuring full efficacy and safety both in vitro and in an animal model (Husain et al., 2015). It remains 
difficult to determine when the "leap of faith" should be taken. Ensuring the proper edit each and every time is essential if researchers are to move into experimental clinical trials and general use of the technology.

Mosaicism, the unintended editing of some but not all cells leading to cells being genetically different, remains as one of the most devastating consequences of improper editing. The overall CRISPR-Cas9 system has not been studied heavily which often causes guesswork at the rate and timing of editing as well as determining whether or not each cell receives the edit (Page, 2017). It is important to ensure the correct and complete placement of the edit to prevent mosaicism, the dangerous result of cells dividing before the intended editing is able to finish. This results in daughter cells that are either not correctly edited or are not edited at all and carry on as they were before the edit (Reyes \& Lanner, 2017). Problems arise as this introduces changes to the cells we cannot control or predict; the edits can be either mono-allelic or bi-allelic. Genetic change needs to occur on both alleles; if it is mono-allelic the change is only made on one allele of the chromosome, while bi-allelic changes are made on both alleles of the chromosome. Incomplete editing causes the genome to be split on which sequence to read, causing problems in the gene product that is produced and the eventual pathway it acts on (Talukder et al., 2016). Furthermore, mono-allelic edits lead to problems of incongruence within the cell and possible mutations being introduced due to incomplete editing.

\section{Hesitation to Use CRISPR-Cas9}

With over 10,000 genetic disorders each being based on one change in the genome, the need for cures or remedies is necessary (Genes and Human Disease, 2010). In the past, the method of action has often been to treat the symptoms rather than treat the underlying cause. However, with genetic editing, it seems that the reality of treating the underlying cause is soon possible. If there is a mutation, researchers hope to edit the abnormality and fix the problem. However, there are problems that arise with this approach. One hindrance is that editing every cell containing the abnormality is challenging for researchers. If editing cells within a small sector of the body such as liver cells or the heart, clinicians are able to monitor the edits with more precision due to fewer cells needing to be monitored. Conversely, if an abnormality affects every cell in the body, it may be hard to ensure that the editing is fully effective as each cell cannot be monitored individually.

It is difficult for researchers to determine when edits should ethically stop. Correcting any genetic abnormality by editing the genome is logical but brings up problems when people use the technology in an unethical manner. Unlimited editing in the hands of those who do not abide by international medical laws can lead to designer babies, eugenics, and unintended genetic abnormalities (Reyes \& Lanner, 2017). Furthermore, it is important to question if eradicating all genetic disorders is ethical. Eradicating genetic disorders and making this a priority pushes the idea that those with genetic disorders are less than those without. Seeking to have the option of choice is more important in shaping the dialogue. Some families may choose to edit the genome of a baby affected by a life threatening genetic disorder rather than abort the fetus. A holistic review of the technology and its consequences is necessary before making long term decisions on what CRISPR can be used for. It is important to ensure that the CRISPR system is in the possession of trained researchers and physicians as licensed and ethical use will curb the negative use of such a powerful technology (Reyes \& Lanner, 2017).

\section{Introduction to Cancer}

It can be argued that within modern medicine there is not a more perplexing group of disorders than cancer (Wanner, n.d.). Cancer is defined as a "group of related disorders in which cells divide uncontrollably and over time spread to other tissues at which time it has 'metastasized"" with the nature of the cells dividing uncontrollably being directly correlated to underlying genetic abnormalities ("Comprehensive Cancer Information", n.d.). Modern technology has increased detection and treatment rates, but the prevalence of cancer has been increasing over the last 50 years. In fact, it is projected that from 2012 to 2030 the incidence will increase 50\% in the United States from 14 million to 21 million and the overall mortality will increase $60 \%$ from 8 million to 13 million with a large part of this being attributed to an aging population. However, the projected statistics are not as morbid considering from 1990 to 2014 the mortality rate decreased $25 \%$ in the United States and is expected to continue on this trend ("Comprehensive Cancer Information", n.d.). However, even as mortality rates decrease, there is progress to be made on treatments seeking to save lives, opening the avenue for genetic editing to be used in future treatment.

Cancerous cells can begin growing anywhere in the body where cells divide. However, areas with a high turnover rate such as the gastrointestinal tract, skin, and breast are at the highest risk for cancerous growth (Pellettieri \& Alvarado, 2007). As damaged cells are replaced, the newly created cells may mutate and grow abnormally. In a similar fashion, cells that die off but are not removed end up with the new cells growing over top, causing tumors to be formed. These tumor can be benign, consisting of cells that are not cancerous, or the tumor can be found to be malignant, consisting of cells that grow uncontrollably and are cancerous. Cancerous cells are not specialized and as a result continue growing without a purpose for the body. This presents a problem when attempting to halt growth, target the cell type, or determining where the cancer will eventually spread to. These cells ignore apoptotic (cell death) signals and can induce normal adjacent cells to form blood vessels creating highly vascularized tumors. Angiogenesis leads to further, easier growth of the tumor as the blood vessels supply the tumor with oxygen, essential nutrients for growth, and remove waste products.

According to the American Cancer Society's report on Family Cancer Syndromes (2018) patients can be predisposed to the formation of cancer based on the genetics of their family, with each individual's cancer being genetically unique. The combination of abnormalities in each cancer is not often the same even from parent to child who have the same type of cancer. Common familial abnormalities often affect patients with Hereditary Breast and Ovarian Cancer (HBOC), Hereditary Non-Polyposis Colorectal Cancer (HNPCC), and other common disorders that share abnormalities in genes such as TP53, PDL1, EGFR, and PTEN (Family Cancer Syndromes, 2018 ). With a myriad of ways for cells to become cancerous, it can be challenging to treat the global disease of cancer as a whole. A more common sense approach that is moving into clinical trials is personalized medicine (Vogenberg et al., 
2010). This approach treats cancers differently based on their underlying genetic cause and the genome of the patient, targeting the cause rather than the side effects of the cancer.

\section{Cancer Beginning and Spreading}

Cancer cells do not respond to treatment as other cells do and as a result of this, researchers are now studying the root cause of the cancer to begin to treat the disease in new ways. Researchers have observed that cancer cells are able to "hide" from the immune system, able to grow without the body being able to respond ("Comprehensive Cancer Information", n.d.). This poses a problem for clinicians as the body is the first and best defense against abnormal growth and pathogens. Researchers are actively making progress and beginning to understand how cancer cells evade the immune system (Tontonoz, 2016). Furthermore, it has been found that tumors can actually "turn down" $\mathrm{T}$ cells by beating them in metabolic competition, essentially stealing away the resources $\mathrm{T}$ cells need to survive (Zhao et al., 2015).

There are three main classes of genetic abnormalities which cause cells to become cancerous: mutants of protooncogenes, tumor suppressor genes, and DNA repair genes. Each class of gene plays a role in maintaining homeostasis in the cells of the body and when they are aberrant, cancerous growth is often induced. Proto-oncogenes are involved in normal cell growth and division. When these genes are altered they become hyperactive and cause cells to grow and survive when they should not (Chial, 2008). Proto-oncogenes when mutated will turn into oncogenes, promoting oncogenesis and uncontrolled levels of growth rather than healthy cell growth. Tumor suppressor genes work in a similar fashion by inhibiting cell growth, ensuring growth does not exceed its limits and turn cancerous. When alterations to these genes occur, cells can begin to grow in an uncontrolled manner (Hardin et al., 2006). DNA repair genes are heavily involved in preventing cancer growth and persistence of mutations. These genes are involved in repairing the DNA when it is damaged by carcinogens, ultraviolet light, random breaks, and transcription. This is important when discussing CRISPR-Cas9 as the repair of a cut region relies on proper functioning of the DNA repair genes. When DNA repair genes do not work properly, cells tend to develop additional mutations in other genes which can then lead to cancer (Romero-Laorden \& Castro, 2017). The original mutations may not be the root cause, not necessarily causing the cancer but directly causing the mutations that then lead to cancer. It is therefore important to classify cancers based on their mutations and to determine the root cause as drugs often act on specific targets, many of which do not vary across organ systems (Kuijjer et al., 2018). Treating the root cause is often a better route than treating the location of the cancer.

A common approach to treating the root cause is to determine the driver of the cancer, which we may be able to suppress. The initial growth of the cancer, the speed of that growth, and how it metastasizes are important to both understand and control. Controlling the speed of growth of the cancer allows more time to respond and treat the cancer. If clinicians can alter the genes which create the cancerous mass we can begin to stop the growth and possibly prepare other cells in the body to fight against these changes. To stop metastasis we need to identify common genes that are mutated and edit these before the mutations occur.

\section{Traditional Cancer Treatments}

Currently, clinicians attempt to either treat the root of cancer by removing all cancerous tissue or treat the symptoms of the cancer. Clinicians and researchers approach locally advanced and metastatic cancers similarly by first controlling its growth and spread, hoping to lessen symptoms and give time to attack the root of the problem, the tumor mass (Understanding Advanced Cancer, 2016). Cancer does not cause death itself, however, the cancer causes organ dysfunction which eventually kills the patient. Cancer is the root cause, organ failure is the effect, and death is the final result. It is essential to create therapeutics that seek the root cause while also alleviating symptoms.

Researchers are developing immunotherapy in which they manipulate the body's immune system and its constituents to attack cancer cells. The basis for most immunotherapy is creating artificial products of the immune system. Monoclonal antibody therapy is the dominant form of immunotherapy; the antibodies bind to cancer cells and signals the immune system to degrade the cell (Scott et al., 2012). The specificity for the cancer cell is determined through the epitope, the component of the antigen which binds the antibody, and the antigenantibody binding affinity. Once attached, the antibody either degrades the cell or acts as a flag alerting the immune system of the presence of the cancer (Scott et al, 2012). The process of creating these therapeutics is time consuming but many are already on the market to treat different forms of cancer such as trastuzumab (Herceptin) which is used to treat breast cancer (Scott et al., 2012).

Adoptive cell transfer attempts to boost the natural ability of T cells, a type of white blood cell that plays a role in immune response, through the injection of cells that have been altered to better respond to the cancer (Rosenberg et al., 2008). Researchers isolate T cells, selecting for those that best respond to the cancer, grow these cells in culture, and then inject the cells back into the body to react against the cancer. This is a common method of treatment because the therapy uses natural $\mathrm{T}$ cells to boost the immune response to the cancer. Another common immunotherapy option is cytokine therapy, small proteins made by cells which communicate with one another to arm the immune system to defend against foreign pathogens (Lee \& Margolin, 2011). The two main cytokines produced are interferons and interleukins, both of which can be used to activate the immune system against the cancerous cells. These activate inflammation, antigen presentation, co-stimulation, and T-cell survival among many other biological activities (Lee \& Margolin, 2011). All three of the aforementioned immunotherapies are based on the assumption that the body's immune system can ward off and fight cancerous cells. The cancer killing power comes from natural defense rather than foreign chemicals and drugs targeted to kill the cancer.

Chemotherapy is used to kill cancerous cells with chemicals by targeting rapidly dividing cells. A problem with this technique is that the chemicals do not differentiate between cancerous cells and normal tissue in the body that is quickly dividing. According to "Chemotherapy Side Effects" published by the American Cancer Society (2016), some side effects from this non-differentiation are hair loss, intestinal inflammation, anemia, and sloughing of skin in the mouth. These symptoms are among the most common causing patients to shave their head, constantly being nauseous, and having little to no energy. These drugs are given intravenously, orally (pill form), 
intrathecal (space between brain and spinal cord), intraperitoneal (body cavity in abdomen), intra-arterial, or topical. This form of treatment is common for its ability to kill cancerous masses quickly but with the side effects being too severe for late stage patients.

Radiotherapy is the use of radiation to target cancerous masses and kill the tissue quickly. Through the use of high doses of radiation the tumor shrinks and is more easily targeted. This works by damaging the DNA of the cell causing the DNA to be beyond repair and the cell dying off (Baskar et al. 2012). External radiation works by aiming a machine which emits radiation towards the cancer. Internal radiation works by having the source of radiation emit itself within the body. The solid form, brachytherapy, is inserted as a seed, ribbon, or capsule and is placed near the tumor, often being used in cancers of the head and neck, breast, cervix, prostate, or eye. The liquid form, systemic therapy, travels through the body to the intended tissue. This radiation is administered by swallowing a drink or is given intravenously. The radioactive material then travels to the target and kills the cancerous cells. This is often used in cancers of the throat and breast. Both forms of radiotherapy are harsh on the body as the goal of the physician is to completely kill the target mass through mutations to its DNA.

Surgery is a common method of treatment which aims to remove the cancer from the body rather than through the use of chemicals, radiation, or using a patient's immune system. The traditional method of cancer surgery is to excise the cancerous mass and then observe margins to ensure the whole tumor is removed. Another method is to debulk the tumor in areas of the body where it is too dangerous to completely cut away the tumor mass (e.g. the brain). This can also be used to ease symptoms of a cancer if the mass puts pressure on an organ such as the brain or the thyroid. Surgical techniques are often used when the tumor mass is large enough to reach and the cancer is not metastatic; the technique seeks to remove the tumor rather than kill the cells.

The most modern approach is targeted therapy; the clinician targets the genetic changes that allow for the cancerous cells to grow, divide, and spread. This works as a combination therapy of each technique mentioned above. This can help the immune system to destroy cancer cells by making sure the cancerous cells cannot hide from the immune system. This therapy can also stop cancer cells from growing by interfering with growth signaling proteins on the cell surface of the tumor cells. In a similar way, the therapy can stop the signaling pathways for the formation of blood vessels in the cancerous mass, an important step in tumor growth (Zhao \& Adjei, 2015). This can also be used to deliver cell killing substances to cancerous cells through monoclonal antibodies conjugated to toxins, chemotherapy, or radiation. Any cell that does not have the specified target will not be affected. Finally, this can work by starving the cancer of hormones by stopping the body from producing certain hormones or preventing the hormones from acting on the cells (Masoud \& Pages, 2017).

A controversial approach to treating cancer is simply waiting it out. As more patients begin to fear the negative consequences of drugs and traditional treatments, they are deciding to wait the diagnosis out. This is especially common in older patients who are at higher risk for negative reactions to many of the treatments and are at higher risk for death from small complications (Cancers After the Age of 75, n.d.). The success rate for those who wait it out is hard to determine but with new treatments making it to the clinic there are more people who will be able to be treated for cancer without the fear of adverse reactions to the drugs.

\section{Progressive Immunotherapy Options}

A prospect for the use of CRISPR-Cas9 against cancer is using the technology to edit immune responses to cancer. Immunotherapy is a method already used for the treatment of cancer but with traditional methods this is done through time consuming research and testing periods. The current research and development is based on the hope of finding a T-cell therapy, antibody, or some other immune molecule which responds well to the cancer. This can be considered intelligent shotgun targeting as there is not a precise target but simply a scatter shot at a therapeutic. The current course of action can be changed with the introduction of genetic editing. One such trial is already under way analyzing how to edit the chimeric antigen receptor (CAR) to the TRAC (T-cell receptor $\alpha$ receptor) locus to redirect $\mathrm{T}$ cells to mediate tumor rejection (Eyquem et al., 2017). Researchers are essentially "teaching" $\mathrm{T}$ cells to recognize the cancer cells they are to target. The promising aspect of this therapy is genetically altering the CAR to precisely target the cancer of interest, being able to coordinate the body's natural defense mechanisms to destroy the cancer cells. This could be used to quickly target and destroy the tumor and in addition boost the immune system following treatment.

Researchers are also looking to edit antibodies. A recent study reported the editing of Sortase, a prokaryotic enzyme, and Flag tags, a target protein, onto the $\mathrm{C}$-terminal (end region) of an antibody without affecting the affinity of the antibody (Khoshnejad et al., 2018). This is a step towards being able to genetically edit the antibody to bind to a specific epitope. If the clinician knows the epitope presenting on the cancer cell, they can then use CRISPR-Cas9 to edit the binding region of the antibody to seek out this epitope. The field of antibody research can, in the near future, be paired with CRISPR to precisely seek out a cancer cell and either destroy it with a conjugated drug or mark it for destruction by other immune reactions.

A more controversial immunotherapy approach is the use of remodeled HIV particles to target cancer. Researchers are modifying the HIV particles to repurpose its inherent potency against the immune system to instead seek out and degrade cancer, labeling the cells "serial killer" cells as they are able to kill over 100,000 cells each (Maude et al., 2014). This works in a similar way to the CAR T cell strategy being used to redirect $\mathrm{T}$ cells to the targeted cancerous mass (Eyquem et al., 2017). The controversial nature stems from how HIV is portrayed in society as a molecule needing to be avoided at all costs. The once deadly viral particle could possibly be used to kill cancer rather than the immune system of a patient.

\section{Editing the Cancerous Mass}

When clinicians genetically edit a patient's cancer, they can attempt to edit the mass itself, but there are complications that arise immediately. When editing a tumor mass there are three approaches to note: treating from outside in, inside out, or editing one cell and letting this then edit the others, no matter the location of the original cell. One main problem with editing the whole tumor is that often the mass consists of cells with different genetic profiles and therefore must be edited 
differently. This poses a problem for researchers as a critical amount of each separate CRISPR portfolio must be present and placed near the tumor for each cancerous cell to be edited. Furthermore, there is not a large amount of research or literature detailing any approach to editing the mass and therefore the general consensus has turned to editing therapeutics to find and kill cancer rather than edit the tumor itself.

\section{Merging CRISPR-Cas9 and Cancer}

The prospect of merging CRISPR-Cas9 system with cancer therapies quickly attracted research groups once the technology was developed. Similar to other genetic technologies such as cloning, the CRISPR system has also seen backlash for how quickly scientists wanted to use the system in humans for unethical use such as editing the germ line of patients (Cyranoski, 2015). As a result of this, many high profile researchers including Dr. Jennifer Doudna, the selfprescribed creator of CRISPR, have called for a moratorium on using the technology in humans (Wade, 2015). Investigating aspects of the human response to this technology is integral to determining safety before using genetic editing in patients. Furthermore, researchers need to investigate the mutations that will be edited, coming to a better understanding of exactly what should be edited. One such endeavor is looking to use CRISPRCas9 to create a Genome-scale CRISPR-Cas9 Knockout (GeCKO) library to identify genes that are essential for cancer cell viability (Shalem et al., 2013). The GeCKO library would purportedly allow researchers to target specific genes when designing treatments.

A benefit of CRISPR-Cas9 is the ability to edit multiple genes quickly in mice models, rather than needing to wait years breeding the correct genome in a mouse (Yang et al., 2013). This serves as proof of concept that mice can be edited using CRISPR as in vivo models of disease. Disorders can be edited into a mouse model and then used to eventually test a CRISPR editing therapy, with researchers then being able to monitor alleviation of symptoms and whether the genetic abnormality is replaced completely. This freedom to create genetically abnormal mouse models in a quick, cheap way allows for streamlined testing of drugs and other therapeutics before testing on humans.

Finally, researchers can induce gene specific cancer to study the efficiency of therapeutics to treat these cancers. One such study moving towards clinical trials is studying Acute Myeloid Leukemia (AML) cells using CRISPR-Cas9. However, the CRISPR-Cas9 system cannot predict somatic mutations which keeps researchers from accurately predicting the future course of the patients AML (Brabetz et al., 2017). Another example is the use of CRISPR to induce gain or loss of function of different genes. The editing combination of the p53, KRAS, and $L K B 1$ sequences has been found to induce adenocarcinoma in mutant mice allowing for direct targeting of this combination of mutations with therapeutics (Platt et al., 2014). Inducing cancer through a specific pathway allows for researchers to study the method of action the cancer cells use to grow and further mutate. With the possibility of being able to turn on and off gene pathways it is helpful to move ahead of the cancer forming and stop the initial growth.

\section{Hesitation to Use CRISPR-Cas9 for Cancer}

Editing the genome before industry standards are established has negative consequences for patients and the overall population. One of the problems we face when dealing with implementing genetic editing is that the change we make is permanent. Unlike other drugs where the patient can be taken off of the drug while the side effects subside, genetic changes do not subside when we remove the CRISPR-Cas9 molecules from the patient. Furthermore, an issue that has become clear with other drugs is that moving from trials in mice to humans is a challenge. The immune, neurological, and target systems do not always respond in the same way in humans as in mice (Tao \& Reese, 2017). This can present newer challenges to researchers as abnormal genetic alterations are harder to predict compared with drug interactions.

Determining what ethical use looks like for the CRISPRCas9 system remains a reason as to why researchers and clinicians are hesitant to move forward with some research and development projects. Determining who has control of the technology and limiting what they can and should edit presents myriad problems not seen in most other biotechnologies. The power of a genetic editing system is troublesome if used for eugenic based projects. However, that same power can be used to eradicate debilitating genetic disorders. Determining if this technology is worth the risk has kept many long term decisions at bay, leading to the moratorium being proposed on human research and further calls for heavy regulation. Further concern underlying access to CRISPR-Cas9 is ensuring protections against special interests and maintaining open access to more than just the wealthy. The wealth gap is already a welldocumented problem in medicine and many worry that genetic editing could also be monopolized leading to the rich being the only population having access which would only further widen the gap between the quality of life of those with money and those without (Newkirk, 2018).

Unethical use of CRISPR-Cas9 in research is already underway as research groups run trials not approved by international medical and ethical law. In places such as China, the United Kingdom, and Sweden, there are already a number of labs editing viable human embryos for research purposes. The proposed mission of each of these labs is to edit the embryos to treat blood borne disorders, study early embryonic development, and an ambitious edit to introduce resistance to HIV (Callaway, 2016). According to differing bioethicists contacted by David Cyranoski (2015), it can be argued that these edits do not cross the line of conventional bioethics. Nonetheless, the question still remains of when the first "CRISPR baby", a developing embryo that is edited heavily to influence trivial traits such as eye color or more competitive enhancements like height or athletic ability, will be born. This will surely reignite the ethical debate surrounding embryonic editing and the determination of when edits become cosmetic rather than medical.

\section{Conclusions and Prospectus}

The purpose for investigating how to edit the genome is to one day improve quality of life by alleviating genetic disorders and predisposition to disease. When used by those with proper scientific, medical, and ethical training, the technology can be used as a new, revolutionary approach to cancer treatment to fix the problems we have faced with past treatments. With the ability of CRISPR-Cas9 to edit the genome, the reality of personalizing medical treatment is near. However, there are three main answers CRISPR-Cas9 must 
provide for its use to revolutionize how we approach cancer care. First, it must be cheap and easy to customize. Second, the system must fix the problems of lengthy treatment schedules and painful side effects that characterize other drugs clinicians have used in the past. Third, the system must be regulated by an international ethics board which offers guidelines for access, cost, and future development of the technology.

First, the CRISPR-Cas9 system must be cheap and easy to customize to a patient. As mentioned before, the CRISPR system has been touted as easy to both use and customize by researchers. With each patient having a unique personal genetic profile and unique cancer genetic profile, there is not one overarching CRISPR-Cas9 cancer drug that will be created. CRISPR-Cas9 acts as the mechanism to which cancer drugs can be created in response to each unique genome. This level of personal medicine demands a high price tag. With early drugs commanding prices of near half of a million dollars, the years of research and search for profits has pushed prices high (Rockoff, 2018). These prices are not feasible for insurance, hospitals, or patients to pay. Moving forward, the price of creating a unique drug must be reflected truthfully in its price. The CRISPR-Cas9 system itself may be cheap to design for a patient, with prices being estimated at $\$ 500$ per target, but the research and development of the system has pushed prices higher so far (Perkel, 2013).

Second, the CRISPR-Cas9 system must fix the problems of long treatment schedules and adverse side effects of current treatment. As mentioned before, many patients face side effects from chemotherapy, radiation, and surgery that adversely affect their lives for long periods of time. CRISPR-Cas9 and genetic editing must be able to fix these issues. In early trials of genetic editing of cancer therapies, it has been found that there is a negative intense bodily response initially to the drug. Over the course of a few days the reaction continues but with proper medical attention the patient is safe. After this they are essentially cancer-free, simply needing to be monitored for future cancerous growth (Maude, 2016). This proof-of-concept experiment demonstrates that genetic editing can treat cancer.

Third and finally, the CRISPR-Cas9 system must have guidelines set out by an international ethics board such as the Human Genome Organization (HUGO) to discourage and control the unethical use of the technology (Human Genome, n.d.).With the promise of genetic editing comes a quick response to use CRISPR-Cas9 to gain an edge on others. Some cite the "CRISPR baby", a genetically engineered baby with a set of physical characteristics chosen by the parents, as being the greatest threat of a eugenics type movement within the technology (Ledford, 2015). Maintaining ethical use will curb the negative uses of such a technology and more importantly will shape the dialogue going forward on how CRISPR-Cas9 will be used, much like how the Asilomar Conference has ensured the ethical use of molecular cloning since its inception (Berg, 2008).

The future of CRISPR-Cas9 and its ethical use in medicine and research depends on proper development of the system and seeking change to the issues the system has. Fixing issues in the off target effects of the sgRNA and Cas9 must be a priority for the system moving forward. Researchers must approach these problems in the same way they have properly developed other biotechnologies, through trial and error and a little bit of luck. Furthermore, moving forward with a technology that has the potential to eradicate genetic disease and disorder, ethical use must be ensured. The future of cancer treatment potentially lies in the development and use of this technology, making it essential to properly study, educate on, and advance the topic of CRISPR-Cas9 genetic editing.

\section{References}

Agtmaal, E. L., André, L. M., Willemse, M., Cumming, S. A., \& Kessel, I. D. (2017). CRISPR/Cas9-Induced (CTG·CAG)n Repeat Instability in the Myotonic Dystrophy Type 1 Locus: Implications for Therapeutic Genome Editing. Molecular Therapy, 25(1), 24-43.

Baskar, R., Lee, K. A., Yeo, R., \& Yeoh, K. (2012). Cancer and Radiation Therapy: Current Advances and Future Directions. International Journal of Medical Sciences, 9(3), 193-199.

Berg, Paul. "Meetings That Changed the World: Asilomar 1975: DNA Modification Secured.” Nature News, Nature Publishing Group, 17 Sept. 2008, www.nature.com/articles/455290a.

Brabetz, O., Alla, V., Angenendt, L., Schliemann, C., \& Berdel, W. E. (2017). RNA-Guided CRISPR-Cas9 System-Mediated Engineering of Acute Myeloid Leukemia Mutations. Molecular Therapy - Nucleic Acids, 6, 243-248.

Callaway, E. (2016, April 19). Gene-editing research in human embryos gains momentum. Retrieved April 05, 2018, from https://www.nature.com/news/geneediting-research-in-human-embryos-gainsmomentum-1.19767

Cancers after the age of 75. (n.d.). Retrieved from https://www.cancersociety.fi/publications/reports/ca ncer-in-finland-2016/cancers-after-the-age-of-75/

Chaplin, D. D. (2003). 1. Overview of the immune response. Journal of Allergy and Clinical Immunology, 111(2).

Charlesworth, C. T., Deshpande, P. S., Dever, D. P., \& Dejene, B. (2018). Identification of Pre-Existing Adaptive Immunity to Cas9 Proteins in Humans. BioRxiv, 1-15.

Chemotherapy Side Effects. (2016, February 15). Retrieved April 03, 2018, from https://www.cancer.org/treatment/treatments-andside-effects/treatmenttypes/chemotherapy/chemotherapy-sideeffects.html

Chen, S., Sun, H., Miao, K., \& Deng, C. (2016). CRISPRCas9: From Genome Editing to Cancer Research. International Journal of Biological Sciences, 12(12), 1427-1436.

Chial, H. (2008). Proto-oncogenes to Oncogenes to Cancer. Nature Education, 1(1):33.

Cho, S. W., Kim, S., Kim, Y., Kweon, J., Kim, H. S., Bae, S., \& Kim, J. (2013). Analysis of Off-Target Effects of CRISPR/Cas-Derived RNA-Guided Endonucleases and Nickases. Genome Research, 24(1), 132-141.

Comprehensive Cancer Information. (n.d.). Retrieved January 15, 2018, from https://www.cancer.gov/

Cong, L., Ran, F. A., Cox, D., Lin, S., Barretto, R., Habib, N., ... Zhang, F. (2013). Multiplex Genome 
Engineering Using CRISPR/Cas Systems. Science, 339(6121), 819-823.

Cyranoski, D. (2015, March 12). Scientists sound alarm over DNA editing of human embryos. Retrieved April 19,2018 , from https://www.nature.com/news/scientists-soundalarm-over-dna-editing-of-human-embryos-1.17110

Eyquem, J., Mansilla-Soto, J., Giavridis, T., Sjoukje J. C. Van Der Stegen, Hamieh, M., Cunanan, K. M., . . . Sadelain, M. (2017). Targeting a CAR to the TRAC locus with CRISPR/Cas9 enhances tumour rejection. Nature, 543(7643), 113-117.

Family Cancer Syndromes. (2018, January 4). Retrieved from https://www.cancer.org/cancer/cancercauses/genetics/family-cancer-syndromes.html

Garneau, J. E., Dupuis, M., Villion, M., Romero, D. A., Barrangou, R., Boyaval, P., . . Moineau, S. (2010). The CRISPR/Cas bacterial immune system cleaves bacteriophage and plasmid DNA. Nature, 468(7320), 67-71.

Geldenhuys, W. J., Mohammad, A. S., Adkins, C. E., \& Lockman, P. R. (2015). Molecular determinants of blood-brain barrier permeation. Therapeutic Delivery, 6(8), 961-971.

Genes and human disease. (2010, December 07). Retrieved April 18, 2018, from http://www.who.int/genomics/public/geneticdisease s/en/index $2 . h t m l$

Griffiths, A. J., Gelbart, W. M., Wessler, S.R., Lewontin, R.C., Suzuki, D.T., \& Miller, J. H. (1999, January 01). Making Recombinant DNA. Retrieved from https://www.ncbi.nlm.nih.gov/books/NBK21407/

Hardin, J., Bertoni, G., \& Becker, W. M. (2006). The World of the Cell (6th Ed.). San Francisco, CA: Benjamin Cummings.

Hsu, P. D., Scott, D. A., Weinstein, J. A., Ran, F. A., Konermann, S., Agarwala, V., ... Zhang, F. (2013). DNA targeting specificity of RNA-guided Cas9 nucleases. Nature Biotechnology, 31(9), 827-832. Retrieved February 25, 2018.

Human Genome Organization (HUGO) International Ltd. HUGO Committee on Ethics, Law and Society. (n.d.). Retrieved from http://www.hugointernational.org/HUGO-CELS

Husain, S. R., Han, J., Au, P., Shannon, K., \& Puri, R. K. (2015). Gene Therapy for Cancer: Regulatory Considerations for Approval. Cancer Gene Therapy, 22(12), 554-563.

Jinek, M., East, A., Cheng, A., Lin, S., Ma, E., \& Doudna, J. (2013). RNA-programmed genome editing in human cells. ELife, 2. Retrieved January 15, 2017.

Khoshnejad, M., Brenner, J. S., Motley, W., Parhiz, H., Greineder, C. F., Villa, C. H., . . Muzykantov, V. R. (2018). Molecular engineering of antibodies for site-specific covalent conjugation using CRISPR/Cas9. Scientific Reports, 8(1). Retrieved April 3, 2018.

Kidiyoor, A. (2018, February 28). The Uncertain Future of CRISPR-Cas9 Technology. Retrieved March 01, 2018, from https://www.biotechniques.com/news/The-
Uncertain-Future-of-CRISPR-Cas9-

Technology/biotechniques-366696.html

Kuijjer, M. L., Paulson, J. N., Salzman, P., Ding, W., \& Quackenbush, J. (2018). Cancer subtype identification using somatic mutation data. British Journal of Cancer, 118, 1492-1501.

Ledford, H. (2015, June 3). CRISPR, the Disruptor. Retrieved March 22, 2018, from https://www.nature.com/new s/crispr-the-disruptor-1.17673

Ledford, H. (2015, October 13). Where in the world could the first CRISPR baby be born? Retrieved April 29, 2018, from https://www.nature.com/news/where-inthe-world-could-the-first-crispr-baby-be-born1.18542

Lee, S., \& Margolin, K. (2011). Cytokines in Cancer Immunotherapy. Cancers, 3(4), 3856-3893. Retrieved April 2, 2018.

Li, Ling \& He, Zhi-Yao \& Wei, Xia-Wei \& Gao, Guang-Ping \& Wei, Yu. (2015). Challenges in CRISPR/CAS9 Delivery: Potential Roles of Nonviral Vectors. Human gene therapy. 26. 452-62. 10.1089/hum.2015.069.

Macdonald, F., Casson, A. G., \& Ford, C. H. (2004). Molecular Biology of Cancer (2nd ed.). London: BIOS Scientific.

Mali, P., Yang, L., Esvelt, K. M., Aach, J., Guell, M., Dicarlo, J. E., . . Church, G. M. (2013). RNA-Guided Human Genome Engineering via Cas9. Science, 339(6121), 823-826. Retrieved February 17, 2017.

Marraffini, L. A., \& Sontheimer, E. J. (2008). CRISPR Interference Limits Horizontal Gene Transfer in Staphylococci by Targeting DNA. Science, 322(5909), 1843-1845. Retrieved April 4, 2017.

Masoud, V., \& Pagès, G. (2017). Targeted therapies in breast cancer: New challenges to fight against resistance. World Journal of Clinical Oncology, 8(2), 120.

Maude, S. L., Frey, N., Shaw, P. A., Aplenc, R., Barrett, D. M., Bunin, N. J. . . Grupp, S. A. (2016). Chimeric Antigen Receptor-Modified T Cells in Chronic Lymphoid Leukemia; Chimeric Antigen ReceptorModified T Cells for Acute Lymphoid Leukemia; Chimeric Antigen Receptor T Cells for Sustained Remissions in Leukemia. New England Journal of Medicine, 374(10), 998-998. Retrieved April 4, 2018.

Mojica, F. J., Ferrer, C., Juez, G., \& Rodríguez-Valera, F. (1995). Long Stretches of Short Tandem Repeats are Present in the Largest Replicons of the Archaea Haloferax mediterranei and Haloferax volcanii and could be involved in Replicon Partitioning. Molecular Microbiology, 17(1), 85-93. Retrieved February 22, 2018.

Mullin, E. (2018, January 22). The first human CRISPR study in the U.S. could begin at any time. Retrieved April 18,2018 , from https://www.technologyreview.com/s/609999/usdoctors-plan-to-treat-cancer-patients-using-crispr/

Newkirk, V. R., II. (2018, January 19). The American HealthCare System Increases Income Inequality. Retrieved April 19, 2018, from 
https://www.theatlantic.com/politics/archive/2018/0 1/health-care-income-inequality-premiumsdeductibles-costs/550997/

Page, M. L. (2017, March 15). Mosaic problem stands in the way of gene editing embryos. Retrieved from https://www.newscientist.com/article/mg23331174400-mosaic-problem-stands-in-the-way-of-geneediting-embryos/

Pellettieri, J., \& Alvarado, A. S. (2007). Cell Turnover and Adult Tissue Homeostasis: From Humans to Planarians. Annual Review of Genetics, 41(1), 83105.

Perkel, Jeffrey M. "Genome Editing with CRISPRs, TALENs and ZFNs." Biocompare, 27 Aug. 2013, www.biocompare.com/Editorial-Articles/144186Genome-Editing-with-CRISPRs-TALENs-andZFNs/.

Platt, R., Chen, S., Zhou, Y., Yim, M., Swiech, L., Kempton, H., . . . Zhang, F. (2014). CRISPR-Cas9 Knockin Mice for Genome Editing and Cancer Modeling. Cell, 159(2), 440-455. Retrieved January 22, 2018.

Ran, F. A., Hsu, P. D., Wright, J., Agarwala, V., Scott, D. A., \& Zhang, F. (2013). Genome engineering using the CRISPR-Cas9 system. Nature Protocols, 8(11), 2281-2308. Retrieved February 13, 2017.

Reardon, S. (2016, June 22). First CRISPR Clinical Trial Gets Green Light from US Panel. Retrieved March 20, 2018, from https://www.nature.com/news/firstcrispr-clinical-trial-gets-green-light-from-us-panel1.20137

Reyes, A. P., \& Lanner, F. (2017). Towards a CRISPR view of early human development: Applications, limitations and ethical concerns of genome editing in human embryos. Development, 144(1), 3-7. Retrieved February 17, 2018.

Rockoff, J. D. (2018, April 26). The Million-Dollar Cancer Treatment: Who Will Pay? Retrieved April 29, 2018, from https://www.wsj.com/articles/themillion-dollar-cancer-treatment-no-one-knowshow-to-pay-for- 1524740401

Romero-Laorden, N., \& Castro, E. (2017). Inherited mutations in DNA repair genes and cancer risk. Current Problems in Cancer, 41(4), 251-264. Retrieved April 2, 2018.

Rosenberg, S. A., Restifo, N. P., Yang, J. C., Morgan, R. A., \& Dudley, M. E. (2008). Adoptive cell transfer: A clinical path to effective cancer immunotherapy. Nature Reviews Cancer, 8(4), 299 308. Retrieved April 2, 2018.

Scott, A. M., Allison, J. P., \& Wolchok, J. D. (2012). Monoclonal Antibodies in Cancer Therapy. Cancer Immunity, 12. Retrieved April 2, 2018.

Shalem, O., Sanjana, N. E., Hartenian, E., Shi, X., Scott, D. A., Mikkelsen, T. S., ... Zhang, F. (2013).
Genome-Scale CRISPR-Cas9 Knockout Screening in Human Cells. Science, 343(6166), 84-87. Retrieved February 25, 2018.

Talukder, A. K., Agarwal, M., Buetow, K. H., \& Denèfle, P. P. (2016). Tracking Cancer Genetic Evolution using OncoTrack. Scientific Reports,6(1).

Tao, L., \& Reese, T. A. (2017). Making Mouse Models That Reflect Human Immune Responses. Trends in Immunology, 38(3), 181-193. Retrieved April 18, 2018.

Tontonoz, M. (2016, March 24). Stealth Mode: How Metastatic Cancer Cells Evade Detection by the Immune System. Retrieved April 02, 2018, from https://www.mskcc.org/blog/steal th-mode-howmetastatic-cancer-cells-evade-detection-immunesystem

Understanding Advanced Cancer, Metastatic Cancer, and Bone Metastasis. (2016, December 15). Retrieved from

https://www.cancer.org/treatment/understandingyour-diagnosis/advanced-cancer/what-is.html

Vogenberg, F. R., Barash, C. I., \& Pursel, M. (2010). Personalized Medicine Part 1: Evolution and Development into Theranostics. Pharmacy \& Therapeutics, 35(10), 560-562. Retrieved April 18, 2018.

Wade, N. (2015, March 19). Scientists Seek Ban on Method of Editing the Human Genome. Retrieved April 04, 2018, from https://www.nytimes.com/2015/03/20/science/biolo gists-call-for-halt-to-gene-editing-technique-inhumans.html?_r=0

Walter, M. (2017, September 25). GRNA-Cas9 [Digital image]. Retrieved April 4, 2018, from https://commons.wikimedia.org/wiki/File:GRNACas9.png

Wanner, M. (n.d.). Why is cancer so difficult to cure? Retrieved April 18, 2018, from https://www.jax.org/news-andinsights/2015/december/why-no-cure-for-cancer

Yang, H., Wang, H., Shivalila, C., Cheng, A., Shi, L., \& Jaenisch, R. (2013). One-Step Generation of Mice Carrying Reporter and Conditional Alleles by CRISPR/Cas-Mediated Genome Engineering. Cell, 154(6), 1370-1379. Retrieved February 12, 2018.

Zhao, E., Maj, T., Kryczek, I., Li, W., Wu, K., Zhao, L., . . . Zou, W. (2015). Cancer mediates effector T cell dysfunction by targeting microRNAs and EZH2 via glycolysis restriction. Nature Immunology, 17(1), 95-103. Retrieved February 23, 2018.

Zhao, Y., \& Adjei, A. A. (2015). Targeting Angiogenesis in Cancer Therapy: Moving Beyond Vascular Endothelial Growth Factor. The Oncologist,20(6), 660-673. 